\title{
Sorafenib improves lipiodol deposition in transarterial chemoembolization of Chinese patients with hepatocellular carcinoma: a long-term, retrospective study
}

\author{
Lin Zheng ${ }^{1}$, Chen-Yang Guo ${ }^{1}$, Cheng-Shi Chen ${ }^{1}$, Jin-Cheng Xiao ${ }^{1}$, Hong-Tao Hu ${ }^{1}$, \\ Hong-Tao Cheng ${ }^{1}$, Deng-Wei Zong ${ }^{1}$, Li Jiang ${ }^{1}$ and Hai-Liang Li ${ }^{1}$ \\ ${ }^{1}$ Department of Radiology, Zhengzhou University Affiliated Cancer Hospital, Zhengzhou 450008, China \\ Correspondence to: Hai-Liang Li, email: cjr.lihailiang@vip.163.com
}

Keywords: lipiodol deposition, hepatocellular carcinoma, TACE, sorafenib, overall survival

Received: August 02, 2016 Accepted: June 08, $2017 \quad$ Published: June 29, 2017

Copyright: Zheng et al. This is an open-access article distributed under the terms of the Creative Commons Attribution License 3.0 (CC BY 3.0), which permits unrestricted use, distribution, and reproduction in any medium, provided the original author and source are credited.

\section{ABSTRACT}

Objective: Though synergy of sorafenib and transarterial chemoembolization (TACE) in hepatocellular carcinoma (HCC) is well discussed in previous reports, association of lipiodol retention by sorafenib addition to TACE with the survival outcomes remain elusive. Therefore, we studied the impact of sorafenib addition to TACE on survival outcomes mediated by lipiodol retention.

Materials and Methods: This is a long-term, retrospective, single-center study using medical records of patients diagnosed with HCC at the Department of Interventional Radiology of Zhengzhou University Affiliated Cancer Hospital (China) between April 2004 and March 2012.

Results: Lipiodol deposition of $>\mathbf{5 0} \%$ was significantly increased in TACE + sorafenib group $(70.87 \%)$ compared to TACE alone group $(45.11 \%)(P=0.0001)$. Significant increase in lipiodol deposition with sorafenib treatment was observed compared to TACE alone group $(O R=0.449, P=0.041)$. The median overall survival in TACE + sorafenib and TACE alone groups were 38 months $[95 \% \mathrm{CI}=9.772-56.228]$ and 31 months $[95 \% \mathrm{CI}=\mathbf{2 1 . 8 5 5 - 4 0 . 1 4 5 ]}$ respectively. Also, the hazard of death was comparatively greater in TACE alone group than TACE + sorafenib group [HR $=1.071]$. Response rate to the therapy significantly increased after sorafenib administration to TACE patients, [compared to TACE alone treatment [69/103 (66.99\%)] vs 55/133 (41.35\%)], $P=0.0001$.

Conclusions: Lipiodol deposition is significantly increased upon sorafenib addition after TACE. However, there was no significant impact of lipiodol deposition on the survival benefits exerted by the synergistic combination and hence, future prospective trails are warranted to validate the findings of this study.

\section{INTRODUCTION}

Hepatocellular carcinoma $[\mathrm{HCC}]$ is the seventh most frequent cancer in males and ninth most common in females, leading to more than 500000 deaths annually throughout the world [1]. China alone accounts for $53.5 \%$ of HCC cases worldwide [2]. Though tumor resection and liver transplant are the current therapeutic approaches employed to treat HCC, these may be restricted to patients with ill preserved liver functions such as, presence of liver cirrhosis, hypertension portal, coagulopathy, increased bilirubin or hepatic dysfunction [3].

Eventually, transarterial chemoembolization [TACE] has gained wide acceptance as standard of care for treating unresectable HCC [4]. Conventional TACE therapy involves intra-arterial delivery of chemotherapeutic agents along with lipiodol followed by injection of embolizing agents like gelatin sponge particles to necrotize the tumor 
tissue [5]. Though lipiodol is used as a chemo drug eluting substance in TACE, it itself has tumor necrotizing ability. Exogenous Lipiodol administered after resection has also shown increased recurrence free survival in HCC patients [6]. Recent reports on meta-analysis of controlled trials on TACE therapy for HCC claimed that, addition of a chemotherapeutic agent did not exert extra benefit to increase the overall survival (OS) compared to the transarterial embolization [TAE] [7]. Previous studies reported that, poor lipiodol retention by the tumor is a major factor affecting OS and time to progression (TTP) associated with TACE therapy. Further, the risk of disease progression in poor lipiodol retention is negatively correlated with increased TACE interventions and degree of lipiodol deposition after the first TACE [8].

The addition of an anti-angiogenic, vascular endothelial growth factor (VEGF) inhibitor sorafenib, to TACE has been experimented in the recent past to increase the OS and TTP in HCC patients. Several studies have reported the efficiency of sorafenib to prolong OS of HCC patients in combination with TACE [9-13]. Moreover, sorafenib was well tolerated and improved OS after resection in Barcelona Clinic Liver Cancer (BCLC) stage C hepatocellular carcinoma [14] and in patients' refractory to TACE [15]. It has also proven efficiency in preventing $\mathrm{HCC}$ recurrences post liver transplantation [16]. Most of all, sorafenib also increases average interval and frequency of TACE, thereby paving high chances for lipiodol retention by the tissue thereby achieving antitumor effect [17].

Consequently, it can be hypothesized that the beneficial effects of sorafenib along with TACE therapy in HCC may be related to the lipiodol deposition. Till date there are no studies documented on the effect of sorafenib on lipiodol deposition. In this study, we have evaluated the lipiodol retention ability of sorafenib and compared the OS and TTP of HCC between TACE alone and TACE + sorafenib treatment groups.

\section{RESULTS}

\section{Demographic characteristics of the patients}

A total of 236 patients were included in the study. Of which, TACE group had 133 patients (males, $n=117$; females, $n=16$ ) and TACE + sorafenib group had 103 patients (males, $n=88$; females, $n=15$ ). Mean age of patients in TACE group was $57.35 \pm 11.88$ years and TACE + sorafenib group was $53.88 \pm 12.25$ years. There were no statistical differences in the serum level of AFP and HbsAg, Eastern Cooperative Oncology Group (ECOG) performance status, BCLC staging, number of tumors and frequency of TACE between the two groups, except for the Child-Pugh class categories A, B and C, where significant difference between the TACE and TACE + sorafenib group was observed $(P<0.0001)$ (Table 1$)$.

\section{Comparison of Lipiodol deposition profile of TACE and TACE + sorafenib groups}

Iodine oil/ Lipiodol deposition was a short-term outcome measured by analyzing the computer tomography (CT) scan images of patients (Figure 1). Iodine oil deposition of $>50 \%$ [8] was significantly increased in TACE + sorafenib group $(70.87 \%)$ compared to TACE alone group $(45.11 \%)(P=0.0001)$ (Table 1$)$. Upon logistic regression analysis of the two groups we found that sorafenib treatment in patients receiving TACE significantly increased lipiodol deposition $(\mathrm{OR}=0.449$, $P=0.041$ ) (Table 2).

\section{Logistic regression analysis of factors associated with Lipiodol deposition}

Logistic regression showed lipiodol deposition was not significantly affected by increase in age and gender $(\mathrm{OR}=1.006, P=0.627$ and $\mathrm{OR}=1.147, P=0.756$ respectively). Whereas, lipiodol deposition was slightly increased with increased number of TACE interventions $(<3$ TACE vs. $\geq 3$ TACE; OR $=0.984)$, less number of tumors $(<3$ vs. $\geq 3$; OR $=0.812, P=0.958)$ and slightly decreased with increased tumor size $(<7 \mathrm{~cm}$ vs $\geq 7 \mathrm{~cm}$; $\mathrm{OR}=1.236, P=0.679$ ). Lipiodol deposit was further decreased with the presence of Child-Pugh $\mathrm{C}$ class $(\mathrm{OR}=1.585, P=0.252)$ and increased with BCLC stage $\mathrm{B}$ over $\mathrm{C}(\mathrm{OR}=0.675, P=0.248)$, ECOG status $(\mathrm{OR}=0.857, P=0.582)$ and HbsAg status $(\mathrm{OR}=0.869$, $P=0.815)$. None of these impacts were statistically significant (Table 2).

\section{Tumor response evaluation according to modified response evaluation criteria in solid tumors (mRECIST)}

Tumor responses were quantified by observing the CT scans of patients of two groups. Responders (complete response $(\mathrm{CR})+$ partial response $(\mathrm{PR}))$ to the treatment were significantly increased after sorafenib administration to TACE patients, [69/103 (66.99\%)] compared to TACE alone treatment $[55 / 133(41.35 \%)], P=0.0001$. None of the patients reached CR in the TACE alone group and majority of the patients developed stable disease. Details of Tumor response evaluated as per mRECIST are shown in Table 3.

\section{Comparison of time to event rates between TACE alone and TACE + sorafenib groups}

Kaplan Meir curves were constructed for OS and TTP determination and are depicted in Figure 2A-2B respectively. 129/236 patients had died at the time of OS data analysis. The median OS of all the remaining 107 patients was 33.1 months [ $95 \% \mathrm{CI}=24.263-41.937]$. The 
Table 1: Baseline characteristics of HCC patients receiving TACE and TACE + sorafenib

\begin{tabular}{|c|c|c|c|}
\hline Baseline characteristics & TACE + sorafenib & TACE & $P$-value \\
\hline \multicolumn{4}{|l|}{ Gender } \\
\hline Male, $n(\%)$ & $88(85.44)$ & 117 (87.97) & 0.5678 \\
\hline Female, $n(\%)$ & $15(14.56)$ & $16(12.03)$ & \\
\hline \multicolumn{4}{|l|}{ Age } \\
\hline Mean $\pm \mathrm{SD}$ [median] & $53.88 \pm 12.25$ & $57.35 \pm 11.88$ & 0.0291 \\
\hline \multicolumn{4}{|l|}{ Child-Pugh class } \\
\hline $\mathrm{A}, n(\%)$ & $45(43.69)$ & $127(96.21)$ & $<0.0001$ \\
\hline $\mathrm{B}, n(\%)$ & $53(51.46)$ & $5(3.79)$ & \\
\hline $\mathrm{C}, n(\%)$ & $5(4.85)$ & $0(0.00)$ & \\
\hline \multicolumn{4}{|l|}{ AFP } \\
\hline$<200, n(\%)$ & $55(53.40)$ & $59(45.04)$ & 0.2041 \\
\hline$>=200, n(\%)$ & $48(46.60)$ & $72(54.96)$ & \\
\hline \multicolumn{4}{|l|}{ ECOG } \\
\hline $0, n(\%)$ & $52(50.48)$ & $75(56.39)$ & 0.484 \\
\hline $1, n(\%)$ & $33(32.03)$ & $58(43.61)$ & \\
\hline $2, n(\%)$ & $18(17.47)$ & $0(0.00)$ & \\
\hline \multicolumn{4}{|l|}{ BCLC stage } \\
\hline $\mathrm{B}, n(\%)$ & $68(66.02)$ & $85(63.91)$ & 0.7364 \\
\hline $\mathrm{C}, n(\%)$ & $35(33.98)$ & $48(36.09)$ & \\
\hline \multicolumn{4}{|l|}{ HbsAg } \\
\hline Negative, $n(\%)$ & $6(5.83)$ & $10(7.52)$ & 0.6078 \\
\hline Positive, $n(\%)$ & $97(94.17)$ & $123(92.48)$ & \\
\hline \multicolumn{4}{|l|}{ Iodine oil deposit } \\
\hline$<50 \%, n(\%)$ & $30(29.13)$ & $73(54.89)$ & 0.0001 \\
\hline$>=50 \%, n(\%)$ & 73 (70.87) & $60(45.11)$ & \\
\hline \multicolumn{4}{|l|}{ Tumor size } \\
\hline$<7 \mathrm{~cm}, n(\%)$ & $65(65.66)$ & $133(100.0)$ & 0.0000 \\
\hline$>=7 \mathrm{~cm}, n(\%)$ & $34(34.34)$ & $0(0.00)$ & \\
\hline \multicolumn{4}{|l|}{ No. of tumors } \\
\hline$<3, n(\%)$ & $58(58.59)$ & $77(58.33)$ & 0.9693 \\
\hline$>=3, n(\%)$ & $41(41.41)$ & 55 (41.67) & \\
\hline \multicolumn{4}{|l|}{ TACE frequency } \\
\hline$<3, n(\%)$ & 67 (65.69) & $92(69.17)$ & 0.5712 \\
\hline$>=3, n(\%)$ & $35(34.31)$ & $41(30.83)$ & \\
\hline
\end{tabular}

median OS in TACE + sorafenib and TACE alone groups were 38 months $[95 \% \mathrm{CI}=9.772-56.228]$ and 31 months $[95 \%$ CI $=21.855-40.145]$ respectively. The log rank test did not reveal significant difference between the two groups, $P=0.254$. The hazard of death was comparatively greater in TACE alone group than TACE + sorafenib group $[\mathrm{HR}=1.071 ; P=0.816$, non-significant $]$ as observed in the cox proportional hazard analysis (Table 4). Similarly, the median TTP duration in TACE + sorafenib and TACE alone groups were 5.7 months $[95 \% \mathrm{CI}=4.243-7.157]$ and 6 months [95\% CI $=5.2-6.7]$ respectively. However, the log rank test did not reveal significant difference between the two groups, $P=0.645$. The hazard of disease progression was comparatively greater in TACE alone group than TACE + sorafenib group [HR $=1.329 ; P=0.164$, non-significant] as observed in the cox proportional hazard analysis (Table 4). 
Table 2: Factors affecting lipiodol deposition

\begin{tabular}{lcccc}
\hline Independent variable & $\boldsymbol{P}$-value & OR & $\mathbf{9 5 \%}$ CI \\
\hline Age & 0.627 & 1.006 & 0.983 & 1.029 \\
Gender & 0.756 & 1.147 & 0.483 & 2.722 \\
ECOG & 0.582 & 0.857 & 0.495 & 1.484 \\
Child-Pugh class & 0.252 & 1.585 & 0.721 & 3.486 \\
BCLC & 0.248 & 0.675 & 0.347 & 1.314 \\
HbsAg & 0.815 & 0.869 & 0.268 & 2.817 \\
AFP & 0.111 & 0.631 & 0.358 & 1.112 \\
Tumor size & 0.679 & 1.236 & 0.453 & 3.367 \\
No. of tumors & 0.486 & 0.812 & 0.453 & 1.457 \\
TACE times & 0.958 & 0.984 & 0.532 & 1.820 \\
GROUP & 0.041 & 0.449 & 0.208 & 0.968 \\
Constant & 0.309 & 4.706 & &
\end{tabular}

Dependent variable: lipiodol Deposit $(0=<50 \%, 1=>=50 \%)$.

Independent variable: Gender: $1=$ Male, $2=$ Female; Child-Pugh class: $1=\mathrm{A}, 2=\mathrm{B}, 3=\mathrm{C}$; BCLC: 1 = B, $2=\mathrm{C}$; $\mathrm{sAg}$ : 1 = Positive, 0 = Negative; Group: $1=$ TACE + sorafenib, $2=$ TACE;

AFP: $0=<200,1=>=200$; Tumor size: $0=<7 \mathrm{~cm}, 1=>=7 \mathrm{~cm}$; No. of tumors: $0=<3,1=>=3$;

TACE times: $0=<3,1=>=3$.

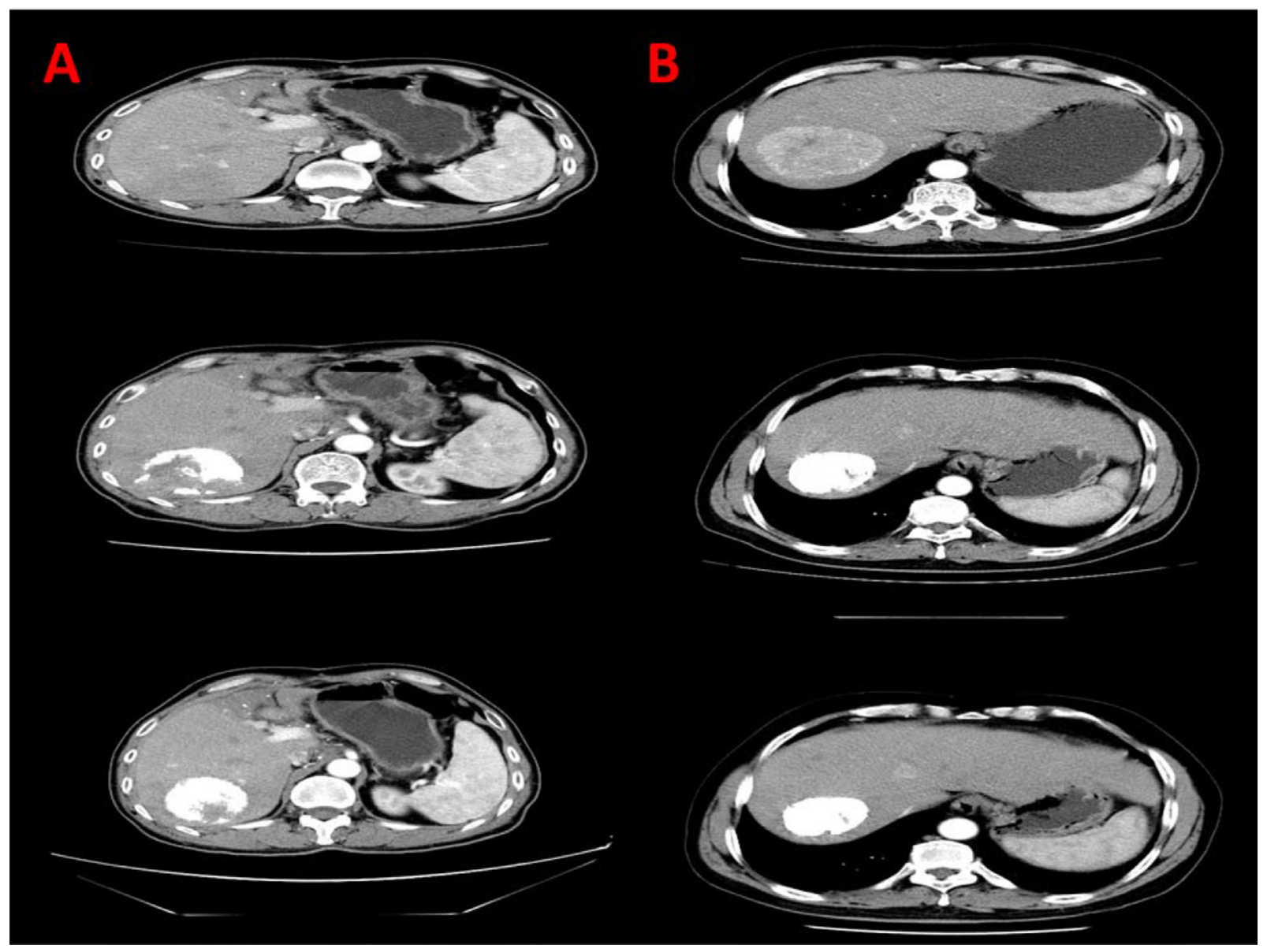

Figure 1: CT scans depiction of lipiodol deposition in TACE alone and TACE + sorafenib group. (A) Lipiodol deposition in the right lobe of liver in TACE alone group. (B) Increased lipiodol deposition in the right lobe of liver in TACE + sorafenib group. 
Table 3: Tumor response evaluation by mRECIST

\begin{tabular}{|c|c|c|c|c|}
\hline MRECIST criteria & $\begin{array}{c}\text { TACE } \\
+ \\
\text { sorafenib }\end{array}$ & TACE & $\chi^{2}$ & $P$ \\
\hline $\mathrm{CR}, n(\%)$ & $2(1.94)$ & $0(0.00)$ & & \\
\hline $\mathrm{PR}, n(\%)$ & $67(65.05)$ & $55(41.35)$ & 15.300 & 0.0001 \\
\hline $\mathrm{SD}, n(\%)$ & $28(27.18)$ & $68(51.13)$ & & \\
\hline $\mathrm{PD}, n(\%)$ & $6(5.83)$ & $10(7.52)$ & & \\
\hline
\end{tabular}

\section{Cox proportional analysis to determine factors associated with OS and TTP}

Cox proportional analysis showed that the hazard of death increased significantly in subjects with BCLC stage $\mathrm{C}$ compared to stage $\mathrm{B}[\mathrm{HR}=1.956 ; P=0.002]$; increase in AFP [HR $=1.491 ; P=0.037]$; and increased number of tumors $[\mathrm{HR}=1.517, P=0.033]$. The hazard significantly decreased in patients with increased TACE interventions $[\mathrm{HR}=0.591, P=0.014]$ and non-significantly with increase in lipiodol oil deposition [HR $=0.981, P=0.923]$. The hazard of disease progression/TTP significantly increased with increased tumor size $[\mathrm{HR}=1.834$, $P=0.017]$. Other factors such as age, gender, ECOG

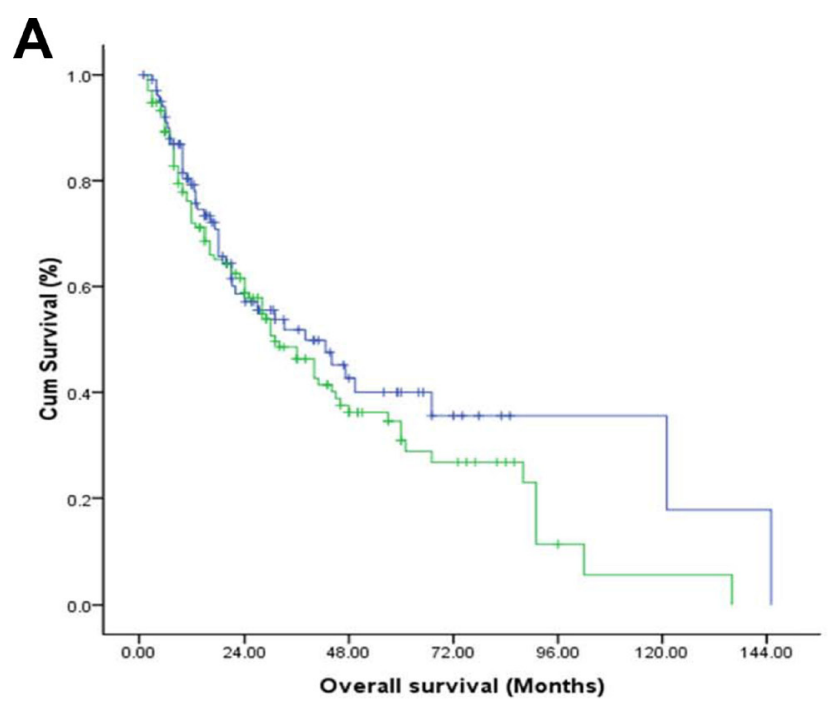

B

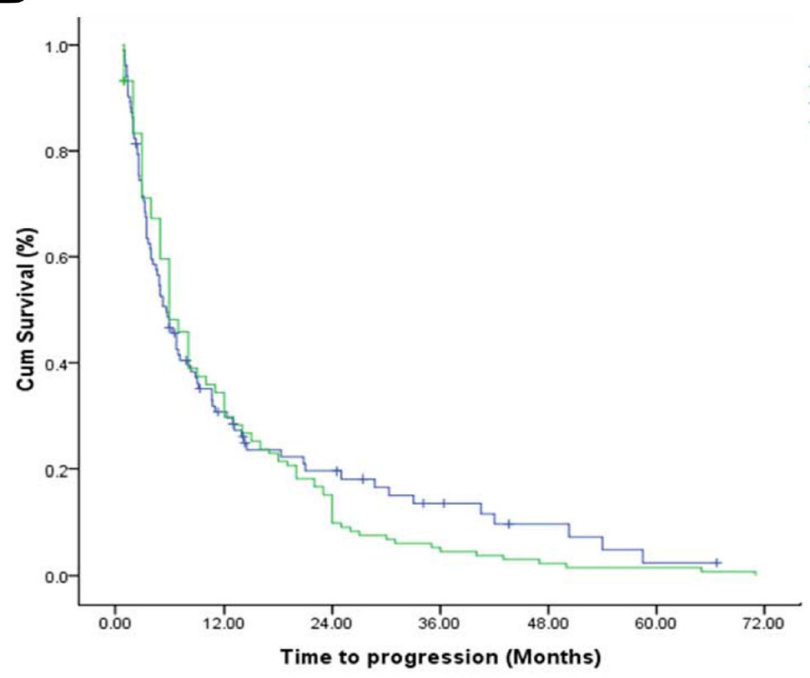

Figure 2: OS and TTP curves of TACE and TACE + sorafenib group. 
Table 4: Cox proportional hazards model OS and TTP

\begin{tabular}{lcccccccc}
\hline \multicolumn{1}{c}{$\begin{array}{c}\text { Independent } \\
\text { variable }\end{array}$} & \multicolumn{9}{c}{ OS } & \multicolumn{1}{c}{ TTP } \\
\cline { 2 - 9 } & $\boldsymbol{P}$-value & HR & \multicolumn{2}{c}{$\mathbf{9 5 \%}$ CI } & $\boldsymbol{P}$-value & HR & 95\% CI \\
\hline Age & 0.354 & 1.008 & 0.991 & 1.024 & 0.630 & 1.003 & 0.992 & 1.014 \\
Gender & 0.622 & 1.164 & 0.637 & 2.127 & 0.226 & 1.317 & 0.844 & 2.055 \\
ECOG & 0.194 & 0.777 & 0.531 & 1.137 & 0.470 & 1.112 & 0.834 & 1.484 \\
Child-Pugh class & 0.108 & 0.633 & 0.363 & 1.105 & 0.873 & 1.035 & 0.677 & 1.583 \\
BCLC & 0.002 & 1.956 & 1.292 & 2.959 & 0.634 & 0.924 & 0.667 & 1.280 \\
HbsAg & 0.188 & 0.632 & 0.320 & 1.251 & 0.530 & 0.832 & 0.468 & 1.478 \\
GROUP & 0.816 & 1.071 & 0.601 & 1.910 & 0.164 & 1.329 & 0.890 & 1.984 \\
Iodine oil deposit & 0.923 & 0.981 & 0.665 & 1.447 & 0.392 & 0.880 & 0.656 & 1.180 \\
AFP & 0.037 & 1.491 & 1.024 & 2.171 & 0.438 & 1.119 & 0.843 & 1.486 \\
Tumor size & 0.005 & 2.485 & 1.310 & 4.715 & 0.017 & 1.834 & 1.115 & 3.016 \\
No. of tumors & 0.033 & 1.517 & 1.033 & 2.227 & 0.138 & 1.254 & 0.930 & 1.692 \\
TACE times & 0.014 & 0.591 & 0.388 & 0.900 & 0.193 & 0.817 & 0.602 & 1.108 \\
\hline
\end{tabular}

Gender: 1 = Male, 2 = Female; Child-Pugh class: 1 = A, 2 = B, 3 = C; BCLC:1 = B, 2 = C; HbsAg: 1 = Positive, 0 = Negative; Group: $1=$ TACE + Sorafenib, $2=$ TACE; Iodine Oil Deposit: $0=<50 \%, 1=>=50 \%$; AFP: $0=<200,1=>=200$; Tumor size: $0=<7 \mathrm{~cm}, 1=>=7 \mathrm{~cm}$; No. of tumors: $0=<3,1=>=3$; TACE times: $0=<3,1=>=3$.

status, Child-Pugh class, HbsAg level had non-significant impact on OS and TTP (Table 4).

\section{Safety events}

Among the major adverse reactions associated with TACE plus sorafenib treatment and TACE alone treatment, occurrence of biloma with abscess was more in TACE treatment group (3 patients, 2.3\%) when compared to TACE-sorafenib group $(1,1 \%$; Table 5$)$. Significant increase in minor adverse events (abdominal pain, fever, vomiting) were observed in TACE alone therapy than TACE-sorafenib combination therapy. In the TACEsorafenib combination treatment, the rate of occurrence of low grade (grade 1-2) sorafenib-related adverse events (hand-foot skin reaction, diarrhea, fatigue, hypertension, rash, alopecia and voice changes) was more. Grade 3 reactions were observed in hand-foot skin reactions $(9(8.7 \%))$ and hypertension $(2(1.9 \%))$ and no grade 4 reactions were reported (Table 6).

\section{DISCUSSION}

In our last retrospective study, TACE effect was proved to be significantly associated with lipiodol retention [8]. Many other studies have also reported that TACE deposition correlates well with tumor necrosis [18-20]. In addition, sorafenib addition to TACE therapy is recommended as an effective therapy for advanced HCC [9-16]. Nevertheless, the underlying mechanism of the synergistic effect were not understood.
HCC is one of the highly-vascularized tumors. Lipiodol is selectively uptaken and retained in hyperarterialyzed liver tumors thereby improving the pharmacokinetics of the drug and tumor response [21]. Circulating VEGF is associated with tumor progression in most of the tumors $[22,23]$. Xin et al. reported that preTACE circulating VEGF levels were significantly high in HCC patients and the levels were considerably decreased post TACE treatment. This further confirms the role of circulating VEGF in HCC also [24]. Moreover, circulating VEGF levels were associated with poor outcomes in patients with diversified tumors [25-28]. Unambiguously, increase in VEGF correlated with decreased lipiodol retention in HCC patients suggesting that VEGF ate up the lipiodol deposited in the tumor [24]. Additionally, in a study conducted to assess the correlation between increased VEGF levels and prognosis after TACE in HCC patients, lipiodol deposition was significantly increased in untreated HCC patients whereas lipiodol deposition increased in 1-2 days after they received first TACE [29]. The reason why we chose iodine oil/lipiodol deposit as the short-term endpoint is due to the assumption that hyper vascular progressing tumor usually washes out deposited lipiodol. As sorafenib can delay tumor progression, the addition of sorafenib to TACE could reduce the consumption of lipiodol. Sorafenib may reduce endothelial nitric oxide synthase (eNOS) activity by inhibiting vascular endothelial growth factor receptors (VEGF-R), leading to a decrease in nitric oxide production and increase lipiodol retention. In the present study, lipiodol has not shown correlation with the survival outcomes 
Table 5: Adverse events related to TACE for the two treatment groups

\begin{tabular}{lcccc}
\hline \multicolumn{1}{c}{ Adverse events } & $\begin{array}{c}\text { TACE }+ \text { sorafenib } \\
\boldsymbol{n}=\mathbf{1 0 3}, \boldsymbol{n}(\mathbf{\%}) \\
\boldsymbol{n} \mathbf{( \% )}\end{array}$ & TACE $\boldsymbol{n}=\mathbf{1 3 3}$ & $\boldsymbol{X}^{2}$ value & $\boldsymbol{P}$-value \\
\hline Major Adverse Events & & & & $1.000^{*}$ \\
Upper GI bleeding & $2(1.9)$ & $2(1.5)$ & & $0.634^{*}$ \\
Biloma with abscess & $1(1.0)$ & $3(2.3)$ & & \\
Minor Adverse Events & & & 0.323 & 0.570 \\
Abdominal pain & $74(71.8)$ & $91(68.4)$ & 0.688 & 0.407 \\
fever & $73(70.9)$ & $98(73.6)$ & 0.493 & 0.482 \\
vomiting & $41(39.8)$ & $59(44.4)$ & & $1.000^{*}$ \\
1-2 myelosuppression & $4(3.8)$ & $5(3.7)$ & \\
\hline
\end{tabular}

TACE, transcatheter arterial chemoembolization; GI, gastrointestinal; "Fisher's Exact Test was used.

Table 6: Incidence of sorafenib-related adverse events in the TACE-sorafenib group

\begin{tabular}{|c|c|c|c|c|}
\hline Adverse events & $\begin{array}{c}\text { All grade, } \\
n(\%)\end{array}$ & $\begin{array}{c}\text { Grade 1-2, } \\
n(\%)\end{array}$ & $\begin{array}{c}\text { Grade 3, } \\
n(\%)\end{array}$ & $\begin{array}{c}\text { Grade 4, } \\
n(\%)\end{array}$ \\
\hline Hand-foot skin reaction & $83(80.6 \%)$ & $74(71.8 \%)$ & $9(8.7 \%)$ & 0 \\
\hline Diarrhea & $66(64.1 \%)$ & $66(64.1 \%)$ & 0 & 0 \\
\hline Fatigue & $25(24.3 \%)$ & $25(24.3 \%)$ & 0 & 0 \\
\hline Hypertension & $9(8.7 \%)$ & $7(6.8 \%)$ & $2(1.9 \%)$ & 0 \\
\hline Rash & $8(7.8 \%)$ & $8(7.8 \%)$ & 0 & 0 \\
\hline Alopecia & $22(21.4 \%)$ & $22(21.4 \%)$ & 0 & 0 \\
\hline Voice changes & $2(1.9 \%)$ & $2(1.9 \%)$ & 0 & 0 \\
\hline
\end{tabular}

associated with the combination treatment, but we find some evidence that increased lipiodol deposition may increase the anti-VEGF activity of sorafenib as lipiodol retention increases the tumor vascular permeability of the drug and hence, increased anti-VEGF activity may be the reason behind the survival benefits. Till date the correlation between lipiodol deposition and sorafenib effect in combination with TACE in HCC is obscure. There is evidence for the antiangiogenic effect mediated efficacy of sorafenib but here we aimed to see if the antiangiogenic property of sorafenib is boosted by increasing the lipiodol retention. As this is a preliminary study on lipiodol retention, we did not show the correlation of lipiodol retention with increased anti-VEGF property and hence a further study on the mechanistic role of lipiodol retention may favor the findings of the present study. The present study findings itself may prove as pioneer evidence for further studies.

Overall our retrospective study revealed a significant increase in lipiodol deposition with addition of sorafenib to TACE compared to TACE alone, proving a synergistic effect of the combination. This may be attributed to the anti-VEGF effect of sorafenib as discussed above. Upon logistic regression, we found that lipiodol deposition was slightly increased with increased number of TACE interventions, decreased number of tumors and BCLC stage B over C, whereas, slightly decreased with increased tumor size, presence of Child-Pugh C class, ECOG and HbsAg status which are in line with our previous reports [8]. None of these impacts were statistically significant, and this may be due to high heterogeneity among the patients and also small sample size and retrospective study nature. Though several meta-analyses of major RCTs showed a superior clinical efficacy of sorafenib addition to TACE therapy over TACE alone therapy, the OS differed tremendously among different patient population, irrespective of the treatment pattern. In a meta-analysis of five comparative studies (2 RCTs) by Wang et al. [30] with a total of 899 patients, though sorafenib increased OS in TACE treated patients the difference was not significant when compared with TACE alone. However, in an earlier meta-analysis of six published studies with a total of 1254 patients a significant difference in OS improvement was seen between the two groups [31]. Nevertheless, a larger meta-analysis on nine studies but with a lower sample size ( $n=900)$, did not yield significant PFS between the groups but however showed a downward trend in progression free rate [32]. From this one may infer that, the response to the treatments vary among patients tremendously and depends on the study design and sample size. Larger sample size 
and RCT design yielded more significant results than other comparative non-RCT studies with smaller sample size. Nevertheless, the non-significant findings had an impact on the clinical efficacy of the treatment. Our study also showed improved OS and TTP in HCC patents, though significant impact of lipiodol deposition on the OS and TTP was not observed. There may be other reasons which may decide the OS and TTP apart from lipiodol deposition in TACE + sorafenib and hence, we restrict our study to the association of lipiodol retention with sorafenib and its synergy with TACE. Also, researchers suggest that TACE plus sorafenib therapy was well tolerated and gave better survival outcomes in advanced HCC patients and hence, as this study involved intermediate stage HCC patients future studies are warranted to delineate the involvement of lipiodol retention on OS and TTP in advanced HCC patients and that the results from this study may be preliminary and an insight that lipiodol retention is improved with sorafenib and further randomized trials are warranted to evaluate the impact of the lipiodol deposition on survival benefits. One more reason for non-significant association of lipiodol deposition with survival may be due to the ethnicity of patients. In previous studies, sorafenib did not significantly prolong TTP or OS in Asian patients compared to non-Asians with $\mathrm{HCC}$ who responded to TACE [33]. Moreover, the incidences of adverse events were also worsened in the patients treated with sorafenib and TACE combination therapy [34]. The complication profile of the therapy reported in previous studies was similar with that of our study. Further, studies by Iavarone et al. [35] and Abou-Alfa [36], reported treatment interruptions and dose changes along with high adverse events in patients who responded to TACE and were treated with sorafenib and that factors such as ECOG status, macrovascular invasion, extrahepatic spread of tumor were independent predictors of survival. Similar way, the lipiodol retention may be influenced by similar predictors and might have led to non-significant association with OS.

To conclude, lipiodol deposition is significantly increased upon sorafenib addition after TACE. However, there was no significant impact of lipiodol deposition on the survival benefits exerted by the synergistic combination and hence, future prospective trails are warranted to validate the findings of this study. Also, the synergy of the combination may be correlated with the anti-VEGF effect of sorafenib which needs further validation.

\section{MATERIALS AND METHODS}

\section{Study design and patient eligibility}

We performed this long-term, retrospective, singlecenter study by evaluating medical records of subjects who were diagnosed with $\mathrm{HCC}$ at the Department of
Interventional Radiology of Zhengzhou University Affiliated Cancer Hospital, China between April 2004 and March 2012. Patients diagnosed with HCC (BCLC stage B or C), Child-Pugh grade A, B or C, ECOG score of 0,1 and 2, those received at least two cycles of TACE assigned to sorafenib (400 mg, twice daily (BID)) group or control group and patients with reported data on iodine oil/lipiodol deposition and efficacy and/or safety analyses were included in the study. Patients previously treated with microwave ablation, radiofrequency ablation, surgical resection or liver transplantation after TACE, platelet count $<50 \times 10^{9} / \mathrm{L}$ were excluded from the study. Participants with missing data were also excluded automatically. Informed consent was not a prerequisite since this was a retrospective study. The patients' information was anonymized throughout the study for confidentiality.

HCC patients were assigned to two cohorts based on the interventions they received, into 1) TACE alone group and; 2) TACE + sorafenib group; in this group sorafenib $400 \mathrm{mg}$ BID was initiated within 1 week before the first TACE. The CT examinations were performed within 1 week of each TACE cycle. The interval between each treatment cycle was 1 month. As lipiodol is retained in the tumor nodules up to 1-year after injection and has also reported immediate retention with a CT examination performed within 7-14 days after TACE [37-39], we performed the CT early for detecting the early retention of lipiodol.

\section{Study outcomes and safety assessment}

Demographic characteristics of patients were obtained from medical records. Primary outcome of the study was to quantify and compare lipiodol deposition in TACE alone and TACE + sorafenib cohorts. Lipiodol deposition was evaluated using CT scans and it was quantified as a measure of increased tumor area density. The denser the tumor area, the maximum is the lipiodol deposition. Secondary outcomes were assessment of OS (defined as the time interval from when the intervention was approved for the patient to death from any cause) and TTP (defined as time from randomization until tumor progression, not including death).

We also examined tumor responses using modified response evaluation criteria in solid tumors (mRECIST) which include CR, disappearance of all clinical evidence of disease), PR (at least 30\% reduction in size of all measurable tumors), stable disease (SD, Between a $30 \%$ reduction or $<25 \%$ increase in the size of all detectable tumors), and progressive disease (PD, Patients or proportion of patients with a $\geq 25 \%$ increase in size of tumors since previous measurement) rates, as the secondary outcome for the patients treated with this combination therapy. Response rate was defined as the proportion of patients with $\mathrm{CR}$ and $\mathrm{PR}$ in the analyzed population. 


\section{Statistical analysis}

Patient demographics, clinical history, laboratory data, and CT scan images were collected. Logistic regression analysis was performed on independent variables that would impact lipiodol deposition included age, gender, ECOG, Child-Pugh class, BCLC stage, $\mathrm{HbsAg}$ and AFP levels, tumor size, number of tumors and frequency of TACE. Descriptive analysis was performed for demographic characteristics and the populations were represented as counts and percentages. KaplanMeier curves were drawn to determine the survival rates (OS) and TTP in subjects of both experimental groups. Cox Proportional Hazard Regression was used for a multivariable adjusted analysis of survival curves with demographic and clinical baseline characteristics. HCC tumor necrosis was measured from CT scan data according to mRECIST criteria. We utilized Chi-square analysis to test association between mRECIST and categorical variables. All statistical analyses were performed with the IBM SPSS statistical software version 22 for Windows (IBM Corp., Armonk, New York, USA). A two-tailed $p$ of $<0.05$ was considered significant.

\section{ACKNOWLEDGMENTS}

We are thankful to Dr. Amit Bhat (PhD) from Indegene Pvt. Ltd (Bangalore, India) for providing medical writing assistance $\&$ critical evaluation of literature during preparation of manuscript.

\section{CONFLICTS OF INTEREST}

The authors have no conflicts of interest to declare.

\section{REFERENCES}

1. World Health Organization. Hepatitis B. http://www.who. int/csr/disease/hepatitis/whocdscsrlyo20022/en/index3.html (accessed on 2/5/16).

2. Al-Mahtab M, Uddin H, Akbar SM. Epidemiology and risk factors of hepatocellular carcinoma in Asia. J Gastroenterol Hepatol Res. 2014; 3:1019-23.

3. Pascual S, Herrera I, Irurzun J. New advances in hepatocellular carcinoma. World J Hepatol. 2016; 8:421-38.

4. Han K, Kim JH. Transarterial chemoembolization in hepatocellular carcinoma treatment: barcelona clinic liver cancer staging system. World J Gastroenterol. 2015; 21:10327-35.

5. Asmis T, Balaa F, Scully L, Papadatos D, Marginean C, Fasih N, Shaw-Stiffel T, Goel R. Diagnosis and management of hepatocellular carcinoma: results of a consensus meeting of The Ottawa Hospital Cancer Centre. Curr Oncol. 2010; 17:6-12.

6. Schwarz L, Bubenheim M, Gardin I, Huet E, Riachi G, Clavier E, Goria O, Vera P, Scotté M. Adjuvant I-131
Lipiodol After Resection or Radiofrequency Ablation for Hepatocellular Carcinoma. World J Surg. 2016; 40:1941-50.

7. Xie ZB, Ma L, Wang XB, Bai T, Ye JZ, Zhong JH, Li LQ. Transarterial embolization with or without chemotherapy for advanced hepatocellular carcinoma: a systematic review. Tumour Biol. 2014; 35:8451-59.

8. Chen CS, Li FK, Guo CY, Xiao JC, Hu HT, Cheng HT, Zheng L, Zong DW, Ma JL, Jiang L, Li HL. Tumor vascularity and lipiodol deposition as early radiological markers for predicting risk of disease progression in patients with unresectable hepatocellular carcinoma after transarterial chemoembolization. Oncotarget. 2016; 7:724152. https://doi.org/10.18632/oncotarget.6892.

9. Lee JM, Jang BK, Lee YJ, Choi WY, Choi SM, Chung WJ, Hwang JS, Kang KJ, Kim YH, Chauhan AK, Park SY, Tak WY, Kweon YO, et al. Survival outcomes of hepatic resection compared with transarterial chemoembolization or sorafenib for hepatocellular carcinoma with portal vein tumor thrombosis. Clin Mol Hepatol. 2016; 22:160-67.

10. Chung YH, Han G, Yoon JH, Yang J, Wang J, Shao GL, Kim BI, Lee TY, Chao Y. Interim analysis of START: study in Asia of the combination of TACE (transcatheter arterial chemoembolization) with sorafenib in patients with hepatocellular carcinoma trial. Int J Cancer. 2013; 132:2448-58.

11. Hsiao WD, Peng CY, Chuang PH, Lai HC, Cheng KS, Chou JW, Chen YY, Yu CJ, Feng CL, Su WP, Chen SH, Kao JT. Evaluation of dose-efficacy of sorafenib and effect of transarterial chemoembolization in hepatocellular carcinoma patients: a retrospective study. BMC Gastroenterol. 2016; 16:50.

12. Geschwind JF, Gholam PM, Goldenberg A, Mantry P, Martin RC, Piperdi B, Zigmont E, Imperial J, Babajanyan S, Foreman PK, Cohn A. Use of Transarterial Chemoembolization (TACE) and Sorafenib in Patients with Unresectable Hepatocellular Carcinoma: US Regional Analysis of the GIDEON Registry. Liver Cancer. 2016; 5:37-46.

13. Geschwind JF, Kudo M, Marrero JA, Venook AP, Chen XP, Bronowicki JP, Dagher L, Furuse J, Ladrón de Guevara L, Papandreou C, Sanyal AJ, Takayama T, Ye SL, et al. TACE Treatment in Patients with Sorafenib-treated Unresectable Hepatocellular Carcinoma in Clinical Practice: final Analysis of GIDEON. Radiology. 2016; 279:630-40.

14. Li J, Hou Y, Cai XB, Liu B. Sorafenib after resection improves the outcome of BCLC stage $\mathrm{C}$ hepatocellular carcinoma. World J Gastroenterol. 2016; 22:4034-40.

15. Arizumi T, Ueshima K, Minami T, Kono M, Chishina $H$, Takita M, Kitai S, Inoue T, Yada N, Hagiwara S, Minami Y, Sakurai T, Nishida N, Kudo M. Effectiveness of Sorafenib in Patients with Transcatheter Arterial Chemoembolization (TACE) Refractory and Intermediate-Stage Hepatocellular Carcinoma. Liver Cancer. 2015; 4:253-62.

16. Sposito C, Mariani L, Germini A, Flores Reyes M, Bongini M, Grossi G, Bhoori S, Mazzaferro V. Comparative efficacy of sorafenib versus best supportive care in recurrent 
hepatocellular carcinoma after liver transplantation: a casecontrol study. J Hepatol. 2013; 59:59-66.

17. Yao X, Yan D, Zeng H, Liu D, Li H. Concurrent sorafenib therapy extends the interval to subsequent TACE for patients with unresectable hepatocellular carcinoma. J Surg Oncol. 2016; 113:672-77.

18. Monsky WL, Kim I, Loh S, Li CS, Greasby TA, Deutsch LS, Badawi RD. Semiautomated segmentation for volumetric analysis of intratumoral ethiodol uptake and subsequent tumor necrosis after chemoembolization. AJR Am J Roentgenol. 2010; 195:1220-30.

19. Takayasu K, Muramatsu Y, Maeda T, Iwata R, Furukawa H, Muramatsu Y, Moriyama N, Okusaka T, Okada S, Ueno $\mathrm{H}$. Targeted transarterial oily chemoembolization for small foci of hepatocellular carcinoma using a unified helical CT and angiography system: analysis of factors affecting local recurrence and survival rates. AJR Am J Roentgenol. 2001; 176:681-88

20. Lee HS, Kim KM, Yoon JH, Lee TR, Suh KS, Lee KU, Chung JW, Park JH, Kim CY. Therapeutic efficacy of transcatheter arterial chemoembolization as compared with hepatic resection in hepatocellular carcinoma patients with compensated liver function in a hepatitis B virus-endemic area: a prospective cohort study. J Clin Oncol. 2002; 20:4459-65.

21. de Baere T, Arai Y, Lencioni R, Geschwind JF, Rilling W, Salem R, Matsui O, Soulen MC. Treatment of Liver Tumors with Lipiodol TACE: Technical Recommendations from Experts Opinion. Cardiovasc Intervent Radiol. 2016; 39:334-43.

22. Kraft A, Weindel K, Ochs A, Marth C, Zmija J, Schumacher P, Unger C, Marmé D, Gastl G. Vascular endothelial growth factor in the sera and effusions of patients with malignant and nonmalignant disease. Cancer. 1999; 85:178-87.

23. Fuhrmann-Benzakein E, Ma MN, Rubbia-Brandt L, Mentha G, Ruefenacht D, Sappino AP, Pepper MS. Elevated levels of angiogenic cytokines in the plasma of cancer patients. Int J Cancer. 2000; 85:40-45.

24. Li X, Feng GS, Zheng CS, Zhuo CK, Liu X. Expression of plasma vascular endothelial growth factor in patients with hepatocellular carcinoma and effect of transcatheter arterial chemoembolization therapy on plasma vascular endothelial growth factor level. World J Gastroenterol. 2004; 10:2878-82.

25. Salven P, Teerenhovi L, Joensuu H. A high pretreatment serum vascular endothelial growth factor concentration is associated with poor outcome in non-Hodgkin's lymphoma. Blood. 1997; 90:3167-72.

26. Salven P, Ruotsalainen T, Mattson K, Joensuu H. High pretreatment serum level of vascular endothelial growth factor (VEGF) is associated with poor outcome in small-cell lung cancer. Int J Cancer. 1998; 79:144-46.

27. Shimada H, Takeda A, Nabeya Y, Okazumi SI, Matsubara H, Funami Y, Hayashi H, Gunji Y, Kobayashi S, Suzuki T, Ochiai T. Clinical significance of serum vascular endothelial growth factor in esophageal squamous cell carcinoma. Cancer. 2001; 92:663-69.

28. Hyodo I, Doi T, Endo H, Hosokawa Y, Nishikawa Y, Tanimizu M, Jinno K, Kotani Y. Clinical significance of plasma vascular endothelial growth factor in gastrointestinal cancer. Eur J Cancer. 1998; 34:2041-45.

29. Shim JH, Park JW, Kim JH, An M, Kong SY, Nam BH, Choi JI, Kim HB, Lee WJ, Kim CM. Association between increment of serum VEGF level and prognosis after transcatheter arterial chemoembolization in hepatocellular carcinoma patients. Cancer Sci. 2008; 99:2037-44.

30. Wang G, Liu Y, Zhou SF, Qiu P, Xu L, Wen P, Wen $J$, Xiao X. Sorafenib combined with transarterial chemoembolization in patients with hepatocellular carcinoma: a meta-analysis and systematic review. Hepatol Int. 2016; 10:501-10.

31. Zhang L, Hu P, Chen $\mathrm{X}$, Bie P. Transarterial chemoembolization (TACE) plus sorafenib versus TACE for intermediate or advanced stage hepatocellular carcinoma: a meta-analysis. PLoS ONE. 2014; 9:e100305.

32. Fu QH, Zhang Q, Bai XL, Hu QD, Su W, Chen YW, Su RG, Liang TB. Sorafenib enhances effects of transarterial chemoembolization for hepatocellular carcinoma: a systematic review and meta-analysis. J Cancer Res Clin Oncol. 2014; 140:1429-40.

33. Kudo M, Imanaka K, Chida N, Nakachi K, Tak WY, Takayama T, Yoon JH, Hori T, Kumada H, Hayashi N, Kaneko S, Tsubouchi H, Suh DJ, et al. Phase III study of sorafenib after transarterial chemoembolisation in Japanese and Korean patients with unresectable hepatocellular carcinoma. Eur J Cancer. 2011; 47:2117-27.

34. Zhong JH. The STORM trial and beyond: narrowing the horizon of adjuvant sorafenib for postoperative hepatocellular carcinoma. Tumour Biol. 2015; 36:8271-72.

35. Iavarone M, Cabibbo G, Piscaglia F, Zavaglia C, Grieco A, Villa $\mathrm{E}$, Cammà $\mathrm{C}$, Colombo $\mathrm{M}$, and SOFIA (SOraFenib Italian Assessment) study group. Field-practice study of sorafenib therapy for hepatocellular carcinoma: a prospective multicenter study in Italy. Hepatology. 2011; 54:2055-63.

36. Abou-Alfa GK. Sorafenib use in hepatocellular carcinoma: more questions than answers. Hepatology. 2014; 60:15-18.

37. Shin SW. The current practice of transarterial chemoembolization for the treatment of hepatocellular carcinoma. Korean J Radiol. 2009; 10:425-34.

38. Golowa YS, Cynamon J, Reinus JF, Kinkhabwala M, Abrams M, Jagust M, Chernyak V, Kaubisch A. Value of noncontrast CT immediately after transarterial chemoembolization of hepatocellular carcinoma with drugeluting beads. J Vasc Interv Radiol. 2012; 23:1031-35.

39. Poyanli A, Rozaneş I, Acunaş B, Sencer S. Palliative treatment of hepatocellular carcinoma by chemoembolization. Acta Radiol. 2001; 42:602-07. 